\begin{tabular}{|c|l|}
\hline Title & HBV reactivation in malignant lymphoma patients treated with rituximab and bendamustine \\
\hline Author(s) & Tsutsumi, Y utaka; Ogasawara, Reiki; Miy ashita, Naohiro; Tanaka, Junji; A saka, Masahiro; Imamura, Masahiro \\
\hline Citation & $\begin{array}{l}\text { International Journal of Hematology, 95(5), 588-591 } \\
\text { https://doi.org/10.1007/312185-012-1050-9 }\end{array}$ \\
\hline Issue Date & 2012-05 \\
\hline Doc URL & http://hdl.handle.net/2115/49380 \\
\hline Rights & The final publication is available at www.springerlink.com \\
\hline Type & article (author version) \\
\hline File Information & IJH95-5_588-591.pdf \\
\hline
\end{tabular}

Instructions for use 


\section{HBV reactivation in malignant lymphoma patients treated with rituximab and bendamustine}

HBV reactivation is a not uncommon complication in patients who receiving chemotherapy with rituximab and steroid. We report two cases in which HBV reactivation occurred after bendamustine treatment combined with rituximab. The first case, a 79-year old female patient, was diagnosed on November 30, 2004 with clinical stage IIIA mantle cell lymphoma, as indicated by swelling of the neck, supraclavicular, and inguinal lymph nodes. Seventy percent R-CHOP was administered from January 7, 2005. At the end of sixth course of R-CHOP, no severe liver damage was detected. At the end of the series of treatments, the lymphoma was considered to be in complete remission, however in July 2006 a recurrence was detected by PET. The patient initially wished to follow a wait-and-see course, but an enlargement was observed in right submaxillary gland in November 2006, and six courses of R-THP-COP therapy $(375 \mathrm{mg} / \mathrm{m} 2$ of rituximab, 1 day; $30 \mathrm{mg} / \mathrm{m} 2$ of Pinorubin, 1 day; $500 \mathrm{mg} / \mathrm{m} 2$ of cyclophosphamide, 1 day; $1 \mathrm{mg} / \mathrm{m} 2$ of vincristine, 1 day; $30 \mathrm{mg} / \mathrm{m} 2$ of Predonine, 5 days) were administered. It was suspected that the lesion remained, at least in part, and radiation therapy was performed $20 \times$ at $40 \mathrm{~Gy}$, leading to a complete remission. Again, no severe liver damage was observed. On August 20, 2008, a recurrence in left infra-axillary lymph node was detected by PET. Radiation therapy was performed 16x at 40 Gy. In May 2009, a recurrence was observed in the hilar area and around abdominal aorta, and in June 2009, treatment (CMR) was started $(0.09 \mathrm{mg} / \mathrm{kg}$ of Cladribine, 5 days; $6 \mathrm{mg} / \mathrm{m} 2$ of mitoxantrone, 1 day; $375 \mathrm{mg} / \mathrm{m} 2$ of rituximab, 1 day). However, protracted myelosuppression continued for four months. In October, three courses of CMR therapy with doses reduced to $20 \%$ were performed, and complete remission was confirmed by $\mathrm{CT}$ and PET. The efficacy of the treatment decreased due to the prolonged interval of treatments caused by protracted myelosuppression, and the patient was elderly, so rituximab was administered every two to three months without Cladribine. In April 2010, invasion of mantle cell lymphoma was found in ileum terminal, indicating a relapse, after which rituximab was administered four times per week, and another dose of rituximab was added once a month. No relapse was observed by CT. In December 2010, however, 
enlargement of left supraclavicular, infra-axillary, and mediastinal lymph nodes was found, and a relapse was confirmed. The patient was hospitalized on January 18, 2011 as the invasion of mantle cells into the gastrointestinal tract had caused diarrhea. After dehydration was improved by transfusion, treatment with rituximab and bendamustine was started on February 1 (375 mg/m2 of rituximab, 1 day; $60 \mathrm{mg} / \mathrm{m} 2$ of bendamustine, 2 days). At that time, HBV-DNA was $2.4 \log$ copy $/ \mathrm{mL}$, HBs antibody was $86.3 \mathrm{mIU} / \mathrm{mL}$, and HBs antigen was negative (These examinations were performed with stored serum, so we suspect that the detected HBV-DNA was a false positive due to contamination). The patent had not received the vaccination. The patient subsequently continued to receive this regimen about once a month. The patient visited the hospital on May 13, 2011 due to general malaise. ALT was increased to $180 \mathrm{IL} / \mathrm{L}, \mathrm{AST}$ was increased to 340 IU/L, and HBs antibody was decreased to $8.3 \mathrm{mIU} / \mathrm{mL}$. HBs antigen was positive, and HBV-DNA was increased to 3.7 log copy/mL (Taqman PCR) (Figure 1). We concluded that this patient had hepatitis due to HBV reactivation, and $0.5 \mathrm{mg}$ of entecavir was administered from May 19, 2011. Liver function returned to normal by June 17; HBVDNA was negative, and HBs antibody recovered to $22.5 \mathrm{mIU} / \mathrm{mL}$. Subsequently, four courses of rituximab and bendamustine were administered, and a complete remission was achieved. Maintenance treatment with rituximab with entecavir is currently ongoing.

The second case is that of a 54-year old female patient who developed follicular lymphoma in a stage IVB right retroperitoneal tumor, as indicated by swelling of the abdominal paraaortic lymph node and invasion of the lumbar vertebra, in June 2006. Six courses of R-CHOP treatment and radiation therapy at the primary site were performed, and a complete remission was achieved. However, a relapse occurred in October 2008, and four courses of CMR therapy were performed. Due to protracted myelosuppression, the dose was reduced to $40 \%$ in the third and fourth courses, after which the patient achieved a complete remission. In 2010, however, the external lymph node on the left cheek was enlarged, and we determined that the patient had relapsed. Although the lesion was reduced by rituximab therapy, new lesions were found bilaterally in orbital regions at the beginning of 2011. Rituximab therapy combined with bendamustine was administered (375 mg/m2 of rituximab, 1 day; $60 \mathrm{mg} / \mathrm{m} 2$ of bendamustine, 2 days). 
Before using rituximab and bendamustine, $\mathrm{HBs}$ antibody was negative, $\mathrm{HBc}$ antibody was positive $(87.8 \mathrm{mIU} / \mathrm{mL}$ ), and HBV-DNA was negative (These were examined in stored serum). The first course of treatment with rituximab and bendamustine was begun on April 11, 2011. The second course was started on May 3, but only a half dose of bendamustine (1 day) was used, due to a urinary-tract infection. Due to protracted myelosuppression and urinary-tract infection, a third course was performed on August 29. Again, a half dose of bendamustine (1 day) was used due to urinary-tract infection. By September 30, AST and ALT had risen to $60 \mathrm{IU} / \mathrm{L}$ and $68 \mathrm{IU} / \mathrm{L}$, respectively. HBs antigen was positive (405 mIU/mL), $\mathrm{HBc}$ antibody was negative $(19.7 \mathrm{mIU} / \mathrm{mL}), \mathrm{HBs}$ antibody was negative $(0.2 \mathrm{mIU} / \mathrm{mL})$, and HBV-DNA was positive $(8.8 \mathrm{log} \mathrm{copy} / \mathrm{mL}$, Taqman PCR). Therefore, we determined that a relapse occurred, and treatment with entecavir, Urso, and glycyrrhizin was started. Liver damage improved rapidly. With chemotherapy before using rituximab and bendamustine, no liver damage occurred, and the follicular lymphoma entered remission.

Since bendamustine was approved in Japan in December 2010, we have performed 18 cases of treatment with rituximab and bendamustine. Of these, five cases were positive for $\mathrm{HBc}$ antibody, and only one case was positive for HBV-DNA, which seemed to be due to contamination. Two of the five cases had HBV reactivation, which means $40 \%$ suffered HBV reactivation. This suggests that HBV reactivation may occur more easily in such regimens, than in treatment with rituximab combined with chemotherapy for HBs antigen-positive cases [1]. These two cases that showed HBV reactivation were summarized in table 1 . Both cases were HBc antibody positive, but case 2 was HBs antibody negative. The case 1 was weak positive for HBV-DNA, but this result was likely to be due to contamination, and the level was at the lowest end of the abnormal range, therefore this case was considered to be negative. Both cases were considered to be HBV-DNA negative before bendamustine treatment. Also, both cases suffered repeated relapses and were administered anti-cancer drugs and rituximab for long periods. This may have caused a condition prone to reactivation, but the effect of bendamustine was not negligible. In these patients, it was necessary to monitor HBV-DNA and examine HBV serology on a monthly basis, but unfortunately these patients were not monitored at 
the initial treatment with rituximab and bendamustine. If they had been monitored properly, HBV reactivation might have been detected earlier.

A growing body of evidence suggests that rituximab treatment and chemotherapy, especially when combined with steroid treatment, may lead to hepatitis due to HBV reactivation [1]. We have also previously reported fluctuations in HBV antibody titer and HBV reactivation [2]. In case 1, a decrease in HBs antibody, an increase in HBV-DNA, and hepatitis development concurrently occurred. Therefore, we determined that the hepatitis was due to HBV reactivation. Also in case 2, we determined that HBV reactivation occurred because $\mathrm{HBc}$ antibody decreased, HBV-DNA increased, and liver damage occurred. However, several questions remain. First, long-term treatment with regimen including steroid was performed first, but HBV reactivation did not occur during that period. Second, the regimen including cladribine but not steroid did not cause hepatitis due to $\mathrm{HBV}$ reactivation, but bendamustine did trigger hepatitis due to HBV reactivation in the cases reported here.

This may be due in part to the fact that rituximab temporarily alters the ratio of $\mathrm{T}$ lymphocytes, which has been already reported. Also, two recent studies have reported that bendamustine itself decreases $\mathrm{T}$ lymphocytes. Therefore, these drugs decreased lymphocyte count, thereby increasing HBV virus [3,4]. None of the cases reported here had CD4 level over 300 counts/uL after bendamustine administration, and in case 2, CD4 level was as low as 49 counts/uL. This CD4 level was similar to that in AIDS, which means that CD4 was low enough to allow HBV proliferation [5]. Another possible reason is that rituximab decreased immunoglobulins generally, resulting in a decrease in antibodies against HBV and enhancement of HBV virus proliferation [2]. Another study mentioned the mechanism of HBV reactivation in a patient treated with bendamustine is B cell depletion, which impairs the antigen presentation and the overreaction of cytotoxic T cell specific responses in HBV reactivation [6]. These may be the reasons that HBV reactivation-induced hepatitis was not severe in either case, probably because the number of lymphocytes was low. In these cases, the count of CD4 before the initial treatment was 
not measured. Performing a CD4 count before the initial treatment might clarify these points.

Fludarabine also suppresses T lymphocytes and tends to cause HBV tolerance, suggesting that drugs that suppress $\mathrm{T}$ lymphocytes could cause the development of hepatitis due to HBV reactivation, even without steroid [7]. There is one case of multiple myeloma that was treated with bendamustine and developed HBV reactivation. There is no report about HBV reactivation in lymphoma [6]. Bendamustine has a purine-analogue structure that caused the effect similar to fludarabine, decreasing lymphocytes, thereby increasing HBV virus, resulting in HBV reactivation. Although we cannot absolutely ignore the effect of prior treatment with cladribine, its administration was terminated over one year ago. We therefore concluded that HBV virus reactivation and hepatitis occured due to 1) decrease in antibody titer by rituximab and 2) fluctuation in $\mathrm{T}$ lymphocytes by bendamustine and rituximab.

Administration of bendamustine or long-term administration of rituximab may cause HBV reactivation even without administration of steroid in patients who did not experience HBV reactivation in the past treatments. Caution should be used for cases positive for $\mathrm{HBc}$ antibody, and preventive administration should be considered.

\section{Figure legends}

Fig. 1

After four courses of treatment with rituximab and bendamustine, the patient was negative for HBs antibody and positive for HBs antigen, and we determined that the patient had developed hepatitis.

1)Manzano-Alonso ML, Castellano Tortajada G. Reactivation of hepatitis B virus infection after cytotoxic chemotherapy or immunosuppressive therapy. World $\mathbf{J}$ Gastroenterol 2011; 17: 1531-1537 
2) Tsutsumi Y, Yamamoto Y, Tanaka J, et al. Prevention of hepatitis B virus reactivation under rituximab therapy. Immunotherapy 2009; 1: 1053-1061

3) Vigna-Perez M, Hernández-Castro B, Paredes-Saharopulos O, Portales-Pérez D, Baranda L, Abud-Mendoza C, \& González-Amaro R. Clinical and immunological effects of Rituximab in patients with lupus nephritis refractory to conventional therapy: a pilot study. Arthritis Research \& Therapy 2006; 8: R83-R92.

4)Klippstein A, Schneider CP, Sayer HG, Hoffken K. Pneumocystis carinii pneumonia as monotherapy in a patient with advanced progressive breast cancer. J Cancer Res Clin Oncol 2003; 129: 316-319

5)Chang JJ, Wightman F, Bartholomeusz A, Ayres A, et al. Reduced hepatitis B virus (HBV)-specific CD4+ T cell responses in human immunodeficiency virus type 1-HBVcoinfected individuals receiving HBV-active antiretroviral therapy. J Virol 2005; 79: 3038-3051

6) Tapan U, May SK, Fiore J, Kozyreva O. Reactivation of hepatitis B virus following bendamustine-containing chemotherapy in a patient with multiple myeloma. Leuk Lymphoma 2011; 52: 916-918

7) Picardi M, Pane F, Quintarelli C, Giudice AD, de Divitiis B, Persico M, Ciancia R, Salvatore F, Rotoli B. Hepatitis B virus reactivation after fludarabine-based regimens for indolent non-Hodgkin's lymphomas: high prevalence of acquired viral genomic mutations. Haematologica 2003; 88: 1296-1303

Yutaka Tsutsumi $^{1}$, Reiki Ogasawara ${ }^{1}$, Naohiro Miyashita ${ }^{1}$, Junji Tanaka ${ }^{2}$, Masahiro Asaka $^{3}$, Masahiro Imamura ${ }^{2}$

${ }^{1}$ Department of Internal Medicine, Hakodate Municipal Hospital, Hakodate, Japan, ${ }^{2}$ Department of Hematology and Oncology, and ${ }^{3}$ Department of Gastroenterology, Hokkaido University Graduate School of Medicine, Sapporo, Japan

Correspondence to: Yutaka Tsutsumi M.D., Department of Internal Medicine, Hakodate Municipal Hospital, 1-10-1, Minato-cho, Hakodate 041-8680, Japan.

Tel: +81-138-43-2000, Fax: +81-138-43-4426

e-mail: yutsutsu@shore.ocn.ne.jp 
Fig 1

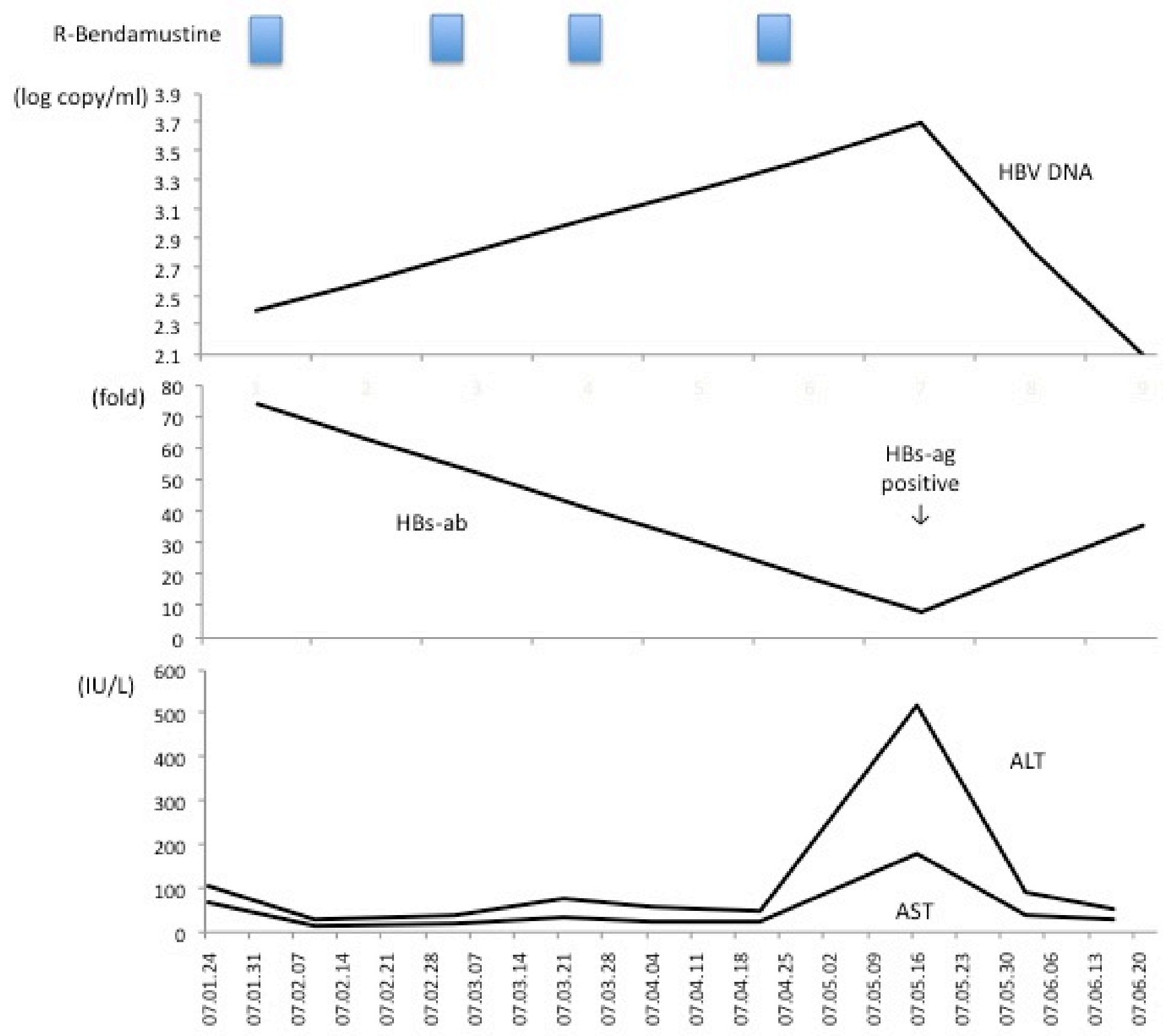


Characteristic of the HBV reactivation in the patients treated with rituimab and bendamusutine

Case 1

Case 2

Age/sex

Diagnose/Disease status

Previous treatment

Outcome of rituximab+bendamustine

Times of treatment

HBs antigen (First/Reactivation)

HBs antibody (First/Reactivation)

$\mathrm{HBc}$ antibody (First/Reactivation)

HBV DNA(Taqman PCR) log copy/ml

(First/Reactivation)

Max AST/ALT in reactivation (IU/L)

HBV treatment

CD4 count after reactivation $(/ \mu \mathrm{L})$

Outcome

Other virus infection
79/Female

$\mathrm{MCL} / 3$ relapse

R-CHOP (70\%), R-THP-COP

+radiation, Radiation, CMR R-

maintanance

$4^{\mathrm{en}} \mathrm{CR}$

4 courses

Negative/positive

Positive(74.2)/Negative(8.3)

Positive(87.2)/Positive(86.9)

$2.4 * / 3.7$

$180 / 340$

Entecavir

292

Alive

VZV
54/Female

$\mathrm{FL} / 2^{\text {id }}$ relapse

R-CHOP+radiation, CMR+R-maintanance, radiation

$3^{\text {a }} \mathrm{CR}$

2 courses

Negative/positive

Negative(0.7)/Negative(0.2)

Positive(87.8)/Negative(19.7)

Negative $/ 8.8$

$60 / 68$

Entecavir

49

Alive

None 